\title{
STORAGE OF MAGNETIC FLUX IN THE OVERSHOOT REGION
}

\author{
F. MORENO-INSERTIS
}

Instituto de Astrofisica de Canarias, 38200 La Laguna (Tenerife), Spain

and

M. SCHÜSSLER and A. FERRIZ-MAS

Kiepenheuer-Institut für Sonnenphysik, Schöneckstr. 6, D-W-7800 Freiburg, Germany

\begin{abstract}
The combined action of the subadiabatic ambient stratification in the overshoot region below the convection zone and the inertial forces associated with the solar rotation is shown to lead to the suppression of the escape of magnetic flux in the form of toroidal flux tubes both toward the surface and toward higher latitudes. We show that a flux ring initially in thermal equilibrium with its environment and rotating with the ambient angular velocity moves radially and latitudinally towards an equilibrium configuration of lower internal temperature and larger internal rotation rate with respect to the surrounding, ficld-frec gas. Wc conclude that flux rings with $B<10^{5} \mathrm{G}$ can be kept within the overshoot region if the superadiabalicity is sufficicntly negative, i.e. $\delta=\nabla-\nabla_{a d} \lesssim-10^{-5}$; below that field strength the poleward drift is also reduced to a latitudinal oscillation of moderate amplitude, $\Delta \theta \lesssim 20 \mathrm{deg}$. Flux rings with significantly larger field strength cannot be kept in the equatorial parts of the overshoot region: their cquilibrium configuration is located at high latitudes far outside the solar activity belts and, at any rate, requires unrealistic values of $\delta$.
\end{abstract}

Key words: Sun: magnetic ficld - stars: magnetic ficld - magnctic fux tubes - dynamo

\section{Introduction}

The possibility of storage of magnetic flux in the form of toroidal flux tubes within the overshoot region at the bottom of the convection zone is limited by two factors. First, if the tubes are close to thermal equilibrium with the surroundings, then they are buoyant and tend to rise. The subadiabatic stratification underneath the convection zone does reduce the density difference between the rising tube and its surroundings, but one has to ascertain if this effect is sufficient to turn the rising motion of the tubes into an oscillation about a position of mechanical equilibrium. This is specially important in view of the results of recent studies which suggest that the field strength of the toroidal flux system from which solar active regions erupt may be significantly larger than equipartition value (for reviews sec MorenoInsertis, 1992, and Schüssler, this volume). Second, the magnetic tension will tend to make the flux rings drift toward the poles, similarly to a rubber band on a polished sphere (poleward slip, Pneuman and Raadu, 1972, Spruit and van Ballegooijen, 1982). Thus, even if their buoyancy could be checked, the tubes would leave the active latitudes on a short timescale (roughly $\varpi / v_{A}$, with $\varpi$ the radius of the ring and $v_{A}$ the Alfvén speed). Yet, as shown below, the poleward slip of the tube can be stopped through the inertial forces associated with the solar rotation: they enforce a latitudinal oscillation of the tube with a frequency which is a simple function of the solar angular velocity.

In this paper we summarize the results of a study of the latitudinal and radial escape of magnetic flux tubes from the active latitudes in the overshoot region. We first show, in the non-rotating case, for which pairs of valucs of ficld strength $B_{0}$ and superadiabatic coefficient $\delta$ (defined as $\nabla-\nabla_{a d}$, with $\nabla=d \log T / d \log P$ and $\nabla_{a d}$ 

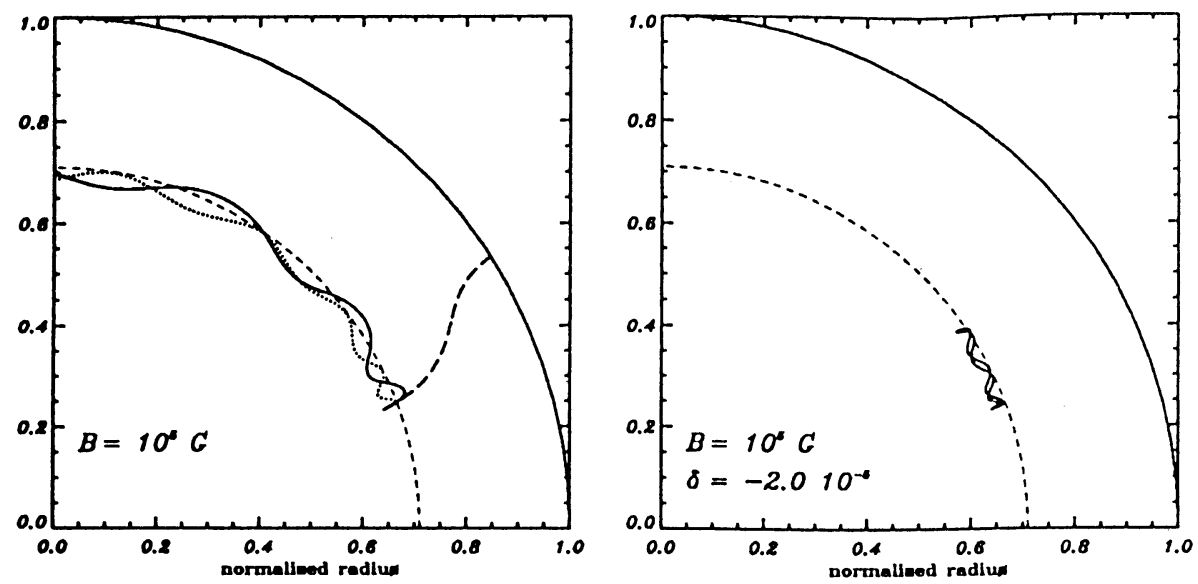

Fig. 1. Trajectories of a mass element of a flux ring in a vertical plane in the absence of rotation (left) and for $\Omega_{e}=2.7 \cdot 10^{-6} \mathrm{sec}^{-1}$ (right). The valucs of $\delta$ chosen in Fig. 1a are $-10^{-5}$ (dashed line), $-1.3 \cdot 10^{-5}$ (solid linc) and $-2 \cdot 10^{-5}$ (dotted linc) .

its adiabatic value) the flux tubes are trapped within the overshoot region. Then we discuss the stabilization of the poleward drift of the tube through rotation. A more complete presentation of the following results can be found in Moreno-Insertis, Schüssler and Ferriz-Mas (1992).

\section{Non-rotating case: vertical trapping and poleward drift.}

We consider flux rings with their symmetry axis pointing along the solar rotation axis. Using the thin flux tube approximation, and given the axial symmetry of the system, the problem at hand thus reduces to the solution of a set of ordinary differential equations which describe the motion of a mass element of the tube subject to buoyancy force, magnetic tension force and (when applicable) the incrtial forces related to rotation. For the convection zone we have chosen Spruit's stratification (Spruit, 1977). For the overshoot region we use a simple polytrope with constant $\delta$, which is then taken as a parameter. Typical trajectories of flux tubes starting from a state of thermal equilibrium with the surroundings in the absence of rotation are depicted in Fig. 1 (a). Shown is the trajectory of a single mass element of the flux tube in a vertical plane (so that the ordinate axis points along the axis of the flux ring) for 3 different values of $\delta$ in the overshoot region, namcly $-10^{-5}$ (dashed line),$-1.3 \cdot 10^{-5}$ (solid line) and $-2 \cdot 10^{-5}$ (dotted linc). In all threc cases $B_{0}=10^{5}$ $\mathrm{G}$, although similar curves can be obtained for other ficld strengths by tuning the value of $\delta$ correspondingly. The small interval in $\delta$ chosen spans the variation between full trapping of the tube in the overshoot region (dotted line) and direct escape to the surface (dashed curve). The two cases with more negative values of $\delta$ execute buoyant oscillations about a state of mechanical equilibrium. In the limit of small amplitude, such oscillations occur with the Brunt-Väisälä frequency (in 
its modified form that incorporates the effect of the magnetic field, non-cartesian geometry, etc, see Moreno-Insertis, Schüssler and Ferriz-Mas (1992). In the same limit, one can easily determine the amplitude of the oscillation of a magnetic ring initially in thermal equilibrium. Conversely, if the calculated amplitude turns out to be larger than the depth of the overshoot region, one can conclude that the tube will escape to the convection zone and rise toward the surface. The values calculated for several pairs $\left(B_{0}, \delta\right)$ are given in Table 1.

\section{TABLE I}

Amplitude (in $\mathrm{km}$ ) of buoyancy oscillation around the position of mechanical equilibrium of a flux tube with field strength $B_{0}$ (in G) initially in thermal equilibrium.

\begin{tabular}{l|lllll} 
& $\delta$ & $-10^{-4}$ & $-10^{-5}$ & $-10^{-6}$ & $-10^{-7}$ \\
$B_{0}$ & & & & & \\
\hline $510^{3}$ & 5.5 & $5.510^{1}$ & $5.510^{2}$ & $5.510^{3}$ \\
$110^{4}$ & $2.210^{1}$ & $2.210^{2}$ & $2.210^{3}$ & $2.310^{4}$ \\
$210^{4}$ & $8.810^{1}$ & $8.810^{2}$ & $8.910^{3}$ & $1.110^{5}$ \\
$110^{5}$ & $2.210^{3}$ & $2.310^{4}$ & $3.710^{5}$ & unstable \\
\hline
\end{tabular}

As can be seen, even strong fields $\left(B_{0} \simeq 10^{5} \mathrm{G}\right)$ can lose a substantial part of their initial buoyancy by rising in the overshoot region if $\delta \lesssim-10^{-5}$. For less negative values of $\delta$, the oscillation amplitudes for strong ficlds will exceed the suggested depth of the subadiabatic region of a few times $10^{4} \mathrm{~km}$ (c.g. al, 1984; Pidatella \& Stix, 1986; Skaley and Stix, 1991 and references therein). In these cases, the flux tube cannot be kept in the overshoot region, leaves the subadiabatic part and rises towards the surface. This will also occur for $B_{0}=10^{5} \mathrm{G}, \delta=-10^{-7}$, for which the corresponding mechanical equilibrium is unstable.

Even if the stratification is subadiabatic enough for the tubes to be trapped at the bottom of the convection zone, the magnetic tension causes the rings to drift towards the pole (as in the trajectories shown by a dashed and a solid line in Fig. 1a), executing buoyant oscillations along the way. A simple way to limit this latitudinal motion is through rotational effects, as scen in the following section.

\section{Effect of rigid rotation}

The dynamical effect of the solar rotation can suppress the poleward drift of the flux tubes and also facilitate their trapping underneath the superadiabatic convection zone. Because of angular momentum conservation, in a rotating coordinate system any motion of the flux ring in the direction transversal to the rotation axis causes an azimuthal flow along the field lines. The Coriolis force associated with this flow in turn effectively opposes the off-axis motion. For instance, for flux tubes in the equatorial plane, the Brunt-Väisälä frequency has, in the rotating case, an additional term $4 \Omega_{e}^{2}$, which, by increasing the frequency of the buoyancy oscillations, reduces their amplitude. Hence, tubes with higher ficld strengths can be trapped in the overshoot region. More importantly, this effect will turn the poleward drift into an oscillatory motion in latitude. For example, assume a flux tube initially in 
thermal equilibrium at an arbitrary latitude $\theta_{1}>0$ and with either high $B_{0}$ or very negative $\delta$ so that the buoyant oscillations have a very small amplitude. In the absence of rotation it will just drift to the pole moving on a sphere of constant radius. In the rotating case, on the contrary, it will execute inertial oscillations of frequency $\omega=2 \Omega_{0} \cos \theta_{0}$ around an equilibrium position at a latitude $\theta_{0}$ given by $\theta_{0}=\theta_{1}\left[1+\left(v_{A 1} / \Omega_{e} r \sin \theta_{1}\right)^{2}\right]^{-1 / 4}$, with $r$ the distance of the ring to the solar center. In a case with arbitrary field strength, the motion will be a superposition of buoyancy and inertial oscillations. This can be seen in Fig. 1b, where the motion of the flux ring is shown for $B_{0}=10^{5} \mathrm{G}, \delta=-2 \cdot 10^{-5}$, having taken $\Omega_{c}=2.7 \cdot 10^{-6}$ $\mathrm{sec}^{-1}$ as the solar angular speed (compare this diagram with the dotted curve of Fig. 1a, corresponding to the same $B_{0}$ and $\delta$ values). A higher field strength (or a less negative value for $\delta$ ) would allow the tube to escape into the convection zone but, because of the rotational forces, it will rise roughly along a direction parallel to the rotation axis (as in Choudhuri and Gilman, 1987).

\section{Conclusions}

Three main conclusions of the foregoing study are:

(a) Rotation facilitates the storage of flux tubes in a convective overshoot region. Flux tubes with a field strength of $10^{5} \mathrm{G}$ which are initially in thermal equilibrium with their environment require $\delta=\nabla-\nabla_{a d} \lesssim-10^{-5}$ in order to be stored. The storage conditions depend also on the thickness of the overshoot layer. Flux tubes with weaker field can be stored in an overshoot region of very small subadiabaticity. Flux tubes of about $10^{4} \mathrm{G}$, for instance, could be kept underneath the convection zone even if the stratification there were strictly adiabatic; the trapping is possible in this case due to the stabilizing effect of rotation.

(b) Outside the equatorial plane, rotation effectively counteracts the tendency of flux tubes to drift towards the pole due to the latitudinal component of the magnetic curvature force. Angular momentum conservation leads to equilibrium configurations with faster internal rotation (so that curvature and rotational forces balance). For field strengths significantly larger than $10^{5} \mathrm{G}$, the equilibrium position is located at high latitudes; this would lead to flux eruption near the poles.

(c) Trapped flux rings perform superposed inertial and buoyancy oscillations about their equilibrium positions. The resulting trajectories cxhibit a Lissajouslike pattern whose orientation depends on the relative importance of rotational, magnetic and stratification effects.

\section{References}

Choudhuri, A.R., Gilman, P.A.: 1987, Astrophys $J$ 316, 788

Moreno-Insertis, F.: 1992, 'The motion of magnetic flux tubes in the convection zone and the subsurface origin of active regions', in Sunspots: Theory and Obscrvations, cd(s). J.H. Thomas and N.O. Weiss, Reidel: Dordrecht, $385-410$.

Moreno-Insertis, F., Schüssler, M., Ferriz-Mas, A.: 1992, Astron. A strophys. 264, $686-700$

Pneuman, G.W., Raadu, M.A.: 1972, Astron. Astrophys. 172, 739

Skaley, D., Stix, M.: 1991, Astron. Astrophys 241, 227

Spruit, H.C.: 1977, Ph.D.Thesis, University of Utrecht.

Spruit, H.C., van Ballegooijen, A.A.: 1982, Astron. Astrophys. 106, 58 